\title{
Previous coronary stents do not increase early and long-term adverse outcomes in patients undergoing off-pump coronary artery bypass grafting: A propensity-matched comparison
}

\author{
Toshihiro Fukui, MD, ${ }^{\mathrm{a}}$ Sachiko Tanaka, MPH, ${ }^{\mathrm{b}}$ and Shuichiro Takanashi, MD ${ }^{\mathrm{a}}$
}

\begin{abstract}
Objective: The aim of our study was to compare the early and long-term outcomes of patients undergoing
\end{abstract} off-pump coronary artery bypass grafting $(\mathrm{CABG})$ with and without previous coronary stents.

\begin{abstract}
Methods: Between September 2004 and September 2011, 269 patients with previous stents underwent first-time isolated off-pump CABG. These patients were compared with 897 patients without previous stent. A propensity score-matching analysis was performed to compare early and late outcomes between the groups. Mean followup time was 43.4 months after surgery.

Results: Patients with previous stents were more likely to be men $(85.9 \%$ in the stent group vs $79.4 \%$ in the no-stent group; $P=.022)$ and more likely to have prior myocardial infarction $(60.2 \%$ vs $36.8 \% ; P<.001)$. Mean number of anastomoses was lower in patients with previous stents than in patients without previous stents (4.0 vs $4.2 ; P=.037$ ). There was no difference in the use of bilateral internal thoracic artery graft between the groups $(88.8 \%$ vs $89.1 \% ; P>.999)$. After propensity adjustment for preoperative characteristics, both operative death $(0.7 \%$ vs $1.5 \% ; P=.414)$ and the major complications rates $(7.8 \%$ vs $7.5 \% ; P=.869)$ were similar between the groups. The actuarial survival rate at 7 years was not different between the groups $(87.2 \% \pm$ $3.2 \%$ vs $84.8 \% \pm 2.9 \% ; P=.470$ ). Furthermore, freedom from major adverse cardiac and cerebrovascular events at 7 years were similar between the groups $(78.9 \% \pm 3.8 \%$ vs $77.6 \% \pm 3.3 \% ; P=.811)$.
\end{abstract}

Conclusions: Previous coronary stents do not increase early and long-term morbidity or mortality in patients undergoing off-pump CABG. (J Thorac Cardiovasc Surg 2014;148:1843-9)

The use of stents during percutaneous coronary intervention (PCI) for the treatment of coronary artery disease has been increasing. However, restenosis is still a major problem after stent implantation. Therefore, the number of patients undergoing coronary artery bypass grafting (CABG) in whom previous PCI procedures have been performed is also increasing. Several investigators have demonstrated that previous PCI with stent is a risk factor of CABG. ${ }^{1-4}$ Massoudy and colleagues ${ }^{3}$ showed in a multicenter analysis that a history of multiple PCIs was an independent predictor for in-hospital deaths and major adverse cardiac events. ${ }^{3}$ In an another multicenter study, Mannacio and colleagues ${ }^{4}$ demonstrated that a history of PCI before CABG increased risk for both operative death and perioperative complications and decreased survival at 5 years' follow-up. Possible reasons for the worse outcome after $\mathrm{CABG}$ in patients with

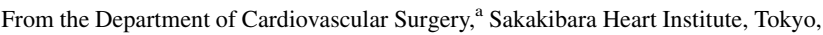
Japan; and the EBM Research Center, ${ }^{b}$ Kyoto University Graduate School of Medicine, Kyoto, Japan.

Disclosures: Authors have nothing to disclose with regard to commercial support.

Received for publication Oct 23, 2013; revisions received Jan 17, 2014; accepted for publication Feb 4, 2014; available ahead of print March 6, 2014.

Address for reprints: Toshihiro Fukui, MD, Department of Cardiovascular Surgery,

Sakakibara Heart Institute, 3-16-1 Asahi-cho, Fuchu City, Tokyo 183-0003, Japan

(E-mail: tfukui.cvs@gmail.com).

$0022-5223 / \$ 36.00$

Copyright (C) 2014 by The American Association for Thoracic Surgery

http://dx.doi.org/10.1016/j.jtcvs.2014.02.004
}

previous stent compared with those without stent include local and systemic inflammatory reactions. ${ }^{5,6}$ Previous studies comparing outcomes in patients with and without previous PCI with stent included CABG using cardiopulmonary bypass, which accelerates systemic inflammatory responses. ${ }^{7}$ Thus we hypothesized that the elimination of cardiopulmonary bypass may diminish the difference in in-hospital and long-term outcomes among patients undergoing CABG with and without previous stent.

The aim of our study was to compare the early and longterm outcomes of patients undergoing off-pump CABG with and without previous coronary stents.

\section{PATIENTS AND METHODS \\ Study Patients}

Between September 2004 and September 2011, 1166 patients underwent first-time isolated off-pump CABG at the Sakakibara Heart Institute. Of those, 269 patients (23.1\%; stent group) had at least 1 previous PCI with stent, whereas 897 patients (76.9\%; no-stent group) underwent Off-pump $\mathrm{CABG}$ as the first-time intervention for the treatment of coronary artery disease. In the stent group, the mean number of PCI procedures with stent per patient was $1.7 \pm 1.2$ in the stent group (single PCI procedure with stent in 162 patients and multiple PCI procedures with stents in 107 patients). Furthermore, the median interval from the last PCI procedure to the CABG operation was 12 months (range, 1 day-254 months). The mean number of implanted stents was $1.8 \pm 1.3$ per patient. Implanted stent material included bare-metal stents in 174 patients, drug-eluting stents in 71 patients, and both in 24 patients. 


\section{Abbreviations and Acronyms \\ $\mathrm{CABG}=$ coronary artery bypass grafting \\ ITA $=$ internal thoracic artery \\ MACCE $=$ major adverse cardiac and cerebrovascular event \\ PCI = percutaneous coronary intervention}

The institutional review board of our institution approved this retrospective study and waived the need for written consent. All data were collected prospectively and reviewed retrospectively. Follow-up involved direct communication with the patient, the patient's family, the attending physician, or a combination of these.

The primary end point of our study was long-term all-cause mortality, including early deaths. The secondary end points were operative death, early major complications, and late major adverse cardiac and cerebrovascular event (MACCE)

\section{Operative Method}

Our operative technique for off-pump CABG has been described previously. ${ }^{8}$ All arterial grafts were harvested in a skeletonized fashion using an ultrasonic scalpel (Harmonic Scalpel, Ethicon Endosurgery, Cincinnati, Ohio). We bypassed all significantly diseased coronary vessels $(\geq 50 \%$ diameter reduction) which were $>1 \mathrm{~mm}$ in diameter. Deep pericardial stay sutures were not used, and a commercially available heart positioner and stabilizer were applied in all cases. We performed extensive reconstruction when the left anterior descending artery was diffusely diseased, and branches such as the septal and diagonal arteries were affected by severe atheromatous plaque. The detailed indications, techniques, and outcomes of extensive reconstruction of the left anterior descending artery have been described previously. ${ }^{9}$

During the operation, graft patency was assessed with a Doppler flow meter in all patients. Furthermore, intraoperative fluorescence imaging has been additionally used since April 2007.

We routinely prescribed low-dose aspirin to all patients postoperatively, continued indefinitely. Clopidogrel was given only in patients with a drugeluting stent.

\section{Definitions}

Nonelective operations included both emergency and urgent cases, according to the definitions in the Society of Thoracic Surgeons database. Operative death was defined as death occurring during the same hospitalization, or after discharge but within 30 days after surgery. Low-output syndrome was defined as the postoperative need for intra-aortic balloon pumping, or any dose of adrenaline, or $>5 \mu \mathrm{g} / \mathrm{kg} / \mathrm{min}$ dopamine or dobutamine. Perioperative myocardial infarction was defined as new $Q$ waves on electrocardiography or a peak creatine kinase MB level of $\geq 5$ times the upper limit of normal (25 IU/L). Respiratory failure was defined as a requirement for prolonged ventilation ( $>48$ hours) or the occurrence of pneumonia. Postoperative stroke was defined as a new neurological deficit that was detected on computed tomography. In patients with preoperative stroke, postoperative stroke was defined as a worsening of the neurologic deficit with new radiologic findings. The major complications included low-output syndrome, perioperative myocardial infarction, respiratory failure, stroke, new requirement of hemodialysis, mediastinitis, and reexploration due to bleeding. MACCE was defined as death from any cause, stroke, myocardial infarction, or repeat revascularization.

\section{Statistical Analysis}

All statistical analyses were performed using the StatView 5.0 software package or the SAS program for Windows, release 9.2 (SAS Institute Inc,
Cary, NC). Continuous variables are reported as the mean \pm standard deviation. Continuous variables were compared by Student $t$ test, whereas discrete variables were compared by the $\chi^{2}$ test or Fischer exact test.

Actuarial survival curves were estimated using the Kaplan-Meier method. The log-rank test was used to assess differences in survival between groups. Multivariate Cox proportional hazards analysis was performed to identify significant predictors of late mortality and late MACCE, using the clinical variables listed in Table 1 and the history of previous stent. Variables with $P<.1$ in univariate analyses were included in the multivariate model.

A propensity score of having previous stent was calculated for each patient using a logistic regression model that included all preoperative variables listed in Table 1. Patients were matched 1:1 on propensity scores with greedy matching techniques. Outcomes of interest between the matched groups were compared using the paired $t$ test for continuous variables, and the McNemar test for discrete variables.

Power analysis for log-rank test indicated that this study has adequate power to detect significant differences for the late death (1- $\beta$ : 0.94 ; survival proportion of 7 years of the no-stent group: 0.84; hazard ratio [HR], 0.27) for an $\alpha$ value of 0.05 .

\section{RESULTS}

\section{Preoperative Characteristics and In-Hospital Outcomes}

Preoperative characteristics of both groups are shown in Table 1. The stent group had higher proportions of men $(P=.022)$ and prior myocardial infarction $(P<.001)$. The mean number of diseased vessels was higher in the no-stent group than in the stent group $(P=.006)$.

The intraoperative and postoperative data are listed in Table 2. The mean number of anastomoses per patient was significantly higher in the stent group than in the nostent group $(P=.037)$. The incidence of performing extensive reconstruction of the left anterior descending artery was higher in the stent group than in the no-stent group $(P=.003)$.

The operative death rates did not differ between these groups $(P>.999)$ and neither did the major complication $(P>$.999). The causes of death were mediastinitis in 1 patient and congestive heart failure in the other patient in the stent group and pneumonia in 6 patients, congestive heart failure in 1 patient, and a rupture of aortic aneurysm in 1 patient in the non-stent group.

\section{Long-Term Outcomes}

Follow-up was complete in 1158 of 1166 patients $(99.3 \%)$. During the follow-up period $(43.4 \pm 24.5$ months), there were 19 deaths in the stent group and 81 deaths in the no-stent group. The actuarial survival rate at 7 years was $87.3 \% \pm 3.2 \%$ in the stent group and $84.4 \%$ $\pm 3.8 \%$ in the no-stent group $(P=.381)$ (Figure $1, A)$. Univariate analyses identified that age, smaller body surface area, acute coronary syndrome, Canadian Cardiovascular Society class III or IV, lower ejection fraction, number of diseased vessel, higher creatinine level, a history of congestive heart failure, peripheral vascular disease, chronic obstructive pulmonary disease, nonelective surgery, and 
TABLE 1. Preoperative characteristics

\begin{tabular}{|c|c|c|c|c|c|c|}
\hline \multirow[b]{2}{*}{ Characteristic } & \multicolumn{3}{|c|}{ Non-matched pairs } & \multicolumn{3}{|c|}{ Propensity-matched pairs } \\
\hline & Stent $(n=269)$ & No-stent $(n=897)$ & $P$ value & Stent $(n=268)$ & No-stent $(\mathrm{n}=\mathbf{2 6 8})$ & $P$ value \\
\hline Age $(y)$ & $68.0 \pm 9.4$ & $68.2 \pm 10.5$ & .786 & $68.0 \pm 9.5$ & $67.3 \pm 11.0$ & .445 \\
\hline Female & $38(14.1)$ & $185(20.6)$ & .022 & $38(14.2)$ & $51(19.0)$ & .128 \\
\hline Body surface area $\left(\mathrm{m}^{2}\right)$ & $1.7 \pm 0.2$ & $1.7 \pm 0.2$ & .882 & $1.7 \pm 0.2$ & $1.6 \pm 0.2$ & .716 \\
\hline Acute coronary syndrome & $94(34.9)$ & $312(34.8)$ & $>.999$ & $93(34.7)$ & $104(38.8)$ & .305 \\
\hline Ejection fraction $(\%)$ & $55.8 \pm 11.3$ & $56.1 \pm 11.8$ & .750 & $55.9 \pm 11.3$ & $56.0 \pm 11.5$ & .862 \\
\hline Diseased vessel & $2.7 \pm 0.5$ & $2.8 \pm 0.4$ & .006 & $2.7 \pm 0.5$ & $2.7 \pm 0.5$ & .718 \\
\hline Left main disease & $91(33.8)$ & $316(35.2)$ & .727 & $91(34.0)$ & $97(36.2)$ & .587 \\
\hline Creatinine (mg/dL) & $1.3 \pm 1.8$ & $1.1 \pm 1.3$ & .100 & $1.3 \pm 1.8$ & $1.3 \pm 1.9$ & .805 \\
\hline Congestive heart failure history & $37(13.8)$ & $121(13.5)$ & .992 & $36(13.4)$ & $32(11.9)$ & .599 \\
\hline Prior myocardial infarction & $162(60.2)$ & $330(36.8)$ & $<.001$ & $161(60.1)$ & $148(55.2)$ & .133 \\
\hline Hypertension & $176(65.4)$ & $629(70.1)$ & .166 & $175(65.3)$ & $171(63.8)$ & .708 \\
\hline Diabetes mellitus & $126(46.8)$ & $419(46.7)$ & $>.999$ & $125(46.6)$ & $130(48.5)$ & .641 \\
\hline Hyperlipidemia & $170(63.2)$ & $572(63.8)$ & .922 & $169(63.1)$ & $170(63.4)$ & .929 \\
\hline Smoking history & $166(61.7)$ & $535(59.6)$ & .592 & $166(61.9)$ & $164(61.2)$ & .862 \\
\hline Previous stroke & $23(8.6)$ & $102(11.4)$ & .230 & $23(8.6)$ & $31(11.6)$ & .228 \\
\hline Peripheral vascular disease & $25(9.3)$ & $68(7.6)$ & .435 & $25(9.3)$ & $19(7.1)$ & .366 \\
\hline Chronic obstructive pulmonary disease & $8(3.0)$ & $34(3.8)$ & .657 & $8(3.0)$ & $2(0.7)$ & .058 \\
\hline Atrial fibrillation & $8(3.0)$ & $49(5.5)$ & .134 & $8(3.0)$ & $9(3.4)$ & .808 \\
\hline
\end{tabular}

Values are presented as mean \pm standard deviation or $\mathrm{n}(\%)$.

atrial fibrillation were associated with late mortality. Multivariate analysis (Table 3) revealed that age, smaller body surface area, lower ejection fraction, higher creatinine level, a history of congestive heart failure, peripheral vascular disease, chronic obstructive pulmonary disease, and atrial fibrillation were independent predictors of late mortality. Previous stent was not identified as an independent predictor of late mortality.
MACCE were observed in 36 patients in the stent group and 140 patients in the no-stent group. Freedom from MACCE at 7 years was $79.1 \% \pm 3.7 \%$ in the stent group and $76.2 \% \pm 2.2 \%$ in the no-stent group $(P=.438)$ (Figure 1, B). Univariate analyses identified that age, smaller body surface area, acute coronary syndrome, Canadian Cardiovascular Society class III or IV, lower ejection fraction, number of diseased vessel, higher

TABLE 2. Operative and postoperative data

\begin{tabular}{|c|c|c|c|c|c|c|}
\hline \multirow[b]{2}{*}{ Variable } & \multicolumn{3}{|c|}{ Non-matched pairs } & \multicolumn{3}{|c|}{ Propensity-matched pairs } \\
\hline & Stent $(\mathbf{n}=\mathbf{2 6 9})$ & No-stent $(n=897)$ & $P$ value & Stent $(n=268)$ & No-stent $(n=268)$ & $P$ value \\
\hline Anastomoses/patient & $4.0 \pm 1.3$ & $4.2 \pm 1.2$ & .037 & $4.0 \pm 1.3$ & $4.2 \pm 1.2$ & .283 \\
\hline Bilateral internal thoracic artery use & $239(88.8)$ & $799(89.1)$ & $>.999$ & $238(88.8)$ & $235(87.7)$ & .691 \\
\hline $\begin{array}{l}\text { Extensive reconstruction of left anterior } \\
\text { descending artery }\end{array}$ & $78(29.0)$ & $182(20.3)$ & .003 & 77 (28.7) & $51(19.0)$ & .012 \\
\hline Operation time (min) & $278.6 \pm 65.0$ & $275.1 \pm 61.1$ & .422 & $278.2 \pm 64.8$ & $269.2 \pm 57.9$ & .010 \\
\hline Intraoperative red blood cell transfusion & $92(34.2)$ & $305(34.0)$ & $>.999$ & $92(34.3)$ & $92(34.3)$ & $>.999$ \\
\hline Intubation (h) & $11.5 \pm 24.8$ & $8.9 \pm 8.3$ & .008 & $11.5 \pm 24.9$ & $9.1 \pm 9.2$ & .091 \\
\hline Intensive care unit stay (d) & $1.7 \pm 3.1$ & $1.9 \pm 8.5$ & .665 & $1.7 \pm 3.1$ & $2.0 \pm 8.3$ & .672 \\
\hline Operative death & $2(0.7)$ & $8(0.9)$ & $>.999$ & $2(0.7)$ & $4(1.5)$ & .414 \\
\hline Major complication & $21(7.8)$ & $69(7.7)$ & $>.999$ & $21(7.8)$ & $20(7.5)$ & .869 \\
\hline Low output syndrome & $4(1.5)$ & $17(1.9)$ & .798 & $4(1.5)$ & $8(3.0)$ & .248 \\
\hline Perioperative myocardial infarction & $2(0.7)$ & $7(0.8)$ & $>.999$ & $2(0.7)$ & $2(0.7)$ & $>.999$ \\
\hline Respiratory failure & $10(3.7)$ & $22(2.5)$ & .368 & $10(3.7)$ & $6(2.2)$ & .314 \\
\hline Severe ventricular arrhythmia & $1(0.4)$ & $9(1.0)$ & .473 & $1(0.4)$ & $3(1.1)$ & .314 \\
\hline Require hemodialysis & $5(1.9)$ & $12(1.3)$ & .737 & $5(1.9)$ & $8(3.0)$ & .405 \\
\hline Stroke & $5(1.9)$ & $12(1.3)$ & .737 & $5(1.9)$ & $2(0.7)$ & .257 \\
\hline Mediastinitis & $1(0.4)$ & $9(1.0)$ & .469 & $1(0.4)$ & 0 & .317 \\
\hline Reexploration due to bleeding & $2(0.7)$ & $10(1.1)$ & .744 & $2(0.7)$ & $2(0.7)$ & $>.999$ \\
\hline Atrial fibrillation & $67(24.9)$ & $233(26.0)$ & .814 & $67(25.0)$ & $74(27.6)$ & .518 \\
\hline
\end{tabular}

Values are presented as mean \pm standard deviation or $\mathrm{n}(\%)$. 

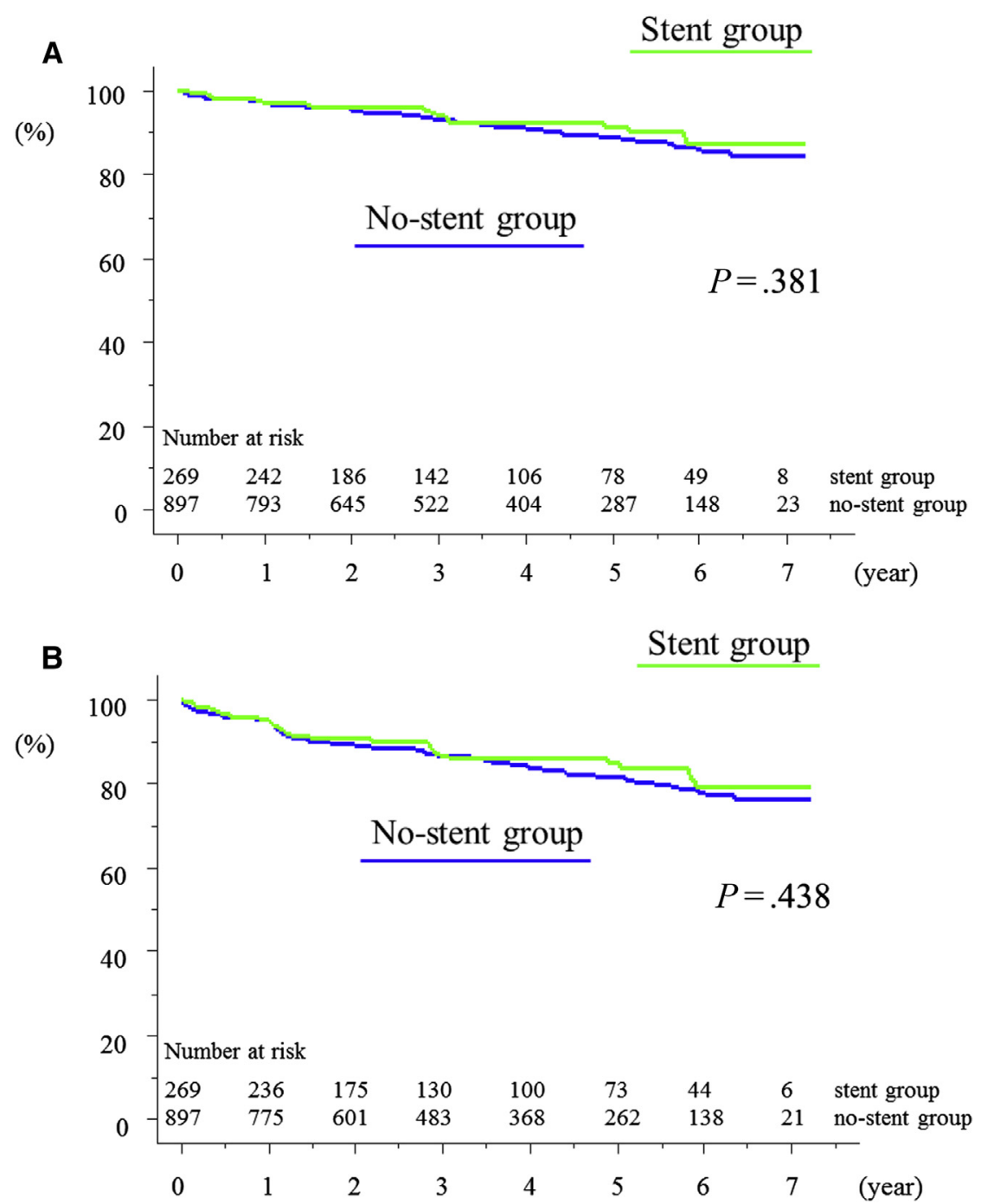

FIGURE 1. A, Kaplan-Meier event-free survival analysis for all deaths in nonmatched patients. B, Kaplan-Meier event-free survival analysis for major adverse cardiac and cerebrovascular events in nonmatched patients.

creatinine level, a history of congestive heart failure, peripheral vascular disease, chronic obstructive pulmonary disease, nonelective surgery, and atrial fibrillation were associated with MACCE. Multivariate analysis (Table 3) revealed that lower ejection fraction, higher creatinine level, peripheral vascular disease, and atrial fibrillation were independent predictors of MACCE. Previous stent was not identified as an independent predictor of MACCE.

\section{Propensity Score Matched-Pairs Analysis}

Preoperative and perioperative data of the propensitymatched patients are listed in Tables 1 and 2. These matched pairs were well balanced for all known covariates (Table 1). The rate of patients undergoing extensive reconstruction of the left anterior descending artery were higher in the stent group than in the no-stent group
$(P=.012)$, which lead to the longer operation time in the stent group than the no-stent group $(P=.010)$.

The operative death rates did not differ between these groups $(P=.414)$ and neither did the major complication rates $(P=.869)$.

The actuarial survival rate at 7 years was not different between the groups $(87.2 \% \pm 3.2 \%$ in the stent group and $84.8 \% \pm 2.9 \%$ in the no-stent group; $P=.470$ ) (Figure 2, A). Furthermore, freedom from MACCE at 7 years was not different between the groups $(78.9 \% \pm$ $3.8 \%$ in stent group and $77.6 \% \pm 3.3 \%$ in the no-stent group; $P=.811$ ) (Figure 2, $B$ ).

\section{DISCUSSION}

Our study demonstrates that previous PCI with stent does not increase early morbidity or mortality in patients undergoing off-pump CABG. Moreover, previous coronary stent 
TABLE 3. Multivariate Cox proportional hazard analysis for late events

\begin{tabular}{|c|c|c|c|}
\hline Outcome & Hazard ratio & $P$ value & $\begin{array}{c}95 \% \text { Confidence } \\
\text { interval }\end{array}$ \\
\hline \multicolumn{4}{|l|}{ Late death } \\
\hline Age & 1.084 & $<.001$ & $1.052-1.116$ \\
\hline Smaller body surface area & 4.878 & .002 & $1.821-13.158$ \\
\hline Lower ejection fraction & 1.016 & .049 & $1.000-1.033$ \\
\hline Creatinine & 1.260 & $<.001$ & $1.156-1.373$ \\
\hline $\begin{array}{l}\text { History of congestive heart } \\
\text { failure }\end{array}$ & 1.764 & .025 & $1.074-2.899$ \\
\hline Peripheral vascular disease & 2.824 & $<.001$ & $1.675-4.762$ \\
\hline $\begin{array}{l}\text { Chronic obstructive } \\
\text { pulmonary disease }\end{array}$ & 2.227 & .010 & $1.212-4.082$ \\
\hline Atrial fibrillation & 2.041 & .025 & $1.096-3.802$ \\
\hline \multicolumn{4}{|l|}{ Late MACCE } \\
\hline Lower ejection fraction & 1.013 & .049 & $1.000-1.027$ \\
\hline Creatinine & 1.119 & .003 & $1.038-1.206$ \\
\hline Peripheral vascular disease & 1.848 & .006 & $1.196-2.857$ \\
\hline Atrial fibrillation & 2.037 & .006 & $1.229-3.367$ \\
\hline
\end{tabular}

MACCE, Major adverse cardiac and cerebrovascular event.

was not an independent predictor of late adverse outcome after off-pump CABG. Similar results have been reported by other investigators. ${ }^{10-12}$ They demonstrated that there were no differences in mortality or morbidity between patients undergoing $\mathrm{CABG}$ with and without previous PCI. ${ }^{10-12}$ Barakate and colleagues ${ }^{10}$ reported similar 30-day mortality rates $(2.0 \%)$ for patients undergoing CABG with and without previous PCI. Van den Brule and colleagues ${ }^{11}$ also described no significant differences in the rates of myocardial infarction, arrhythmia, reinterventions, neurologic complications, renal complications, pulmonary complications, or in-hospital mortality for patients undergoing CABG with and without previous PCI. Furthermore, they reported that a 1-year follow-up did not reveal any significant differences in the rates of cardiacrelated mortality or recurrent ischemic events between their groups. More recently, Boening and colleagues ${ }^{12}$ reported that coronary stenting in patients with diabetes did not predispose to a higher perioperative risk regarding mortality and morbidity after CABG. However, these studies were limited to demonstrate the early outcomes; moreover, CABG was performed with cardiopulmonary bypass in the majority of patients. In our study, we selected patients undergoing off-pump CABG to eliminate the inflammatory effect of cardiopulmonary bypass. Furthermore, long-term outcomes were compared between the groups to investigate the influence of having a stent on patients' prognoses.

In contrast, there are several reports demonstrating that the previous PCI with stent is a risk factor for CABG. Hassan and colleagues ${ }^{2}$ reported that the in-hospital mortality rates after CABG were higher in patients with prior PCI than in patients without prior PCI (3.6\% vs $2.3 \%)$. Furthermore, prior PCI emerged as an independent predictor of postoperative in-hospital mortality. Similarly, Thielmann and colleagues ${ }^{1}$ described that multiple previous PCI procedures were strongly associated with in-hospital mortality (odds ratio, 2.24) and major adverse cardiac events (odds ratio, 2.28). More recently, Massoudy and colleagues ${ }^{3}$ showed in a large multicenter analysis $(\mathrm{N}=37,140)$ that a history of multiple PCIs was an independent predictor for in-hospital death and major adverse cardiac events. Furthermore, Mannacio and colleagues ${ }^{4}$ demonstrated that a history of PCI before CABG decreased survival at 5 years' follow-up. Several negative influences of PCI with stent have been considered. Lazar ${ }^{13}$ suggested that stents not only may result in local vascular inflammation, but also may stimulate platelet and neutrophil adhesion along the entire vessel, with resulting decreased graft patency. Moreover, because of the physical presence of the stent, bypass grafts may need to be inserted more distally in vessels, which may compromise runoff and ultimately patency. ${ }^{10}$ In fact, Gomez and colleagues ${ }^{6}$ found that the coronary artery distal to the stent had chronic inflammation and an intimal acute inflammatory infiltrate with polymorphonuclear leukocytes. Moreover, the myocardium adjacent to the stent had significant chronic inflammatory infiltrate and fibrosis.

The use of cardiopulmonary bypass may amplify these inflammatory reactions both locally and systemically. Then the off-pump procedure may contribute to better outcomes than the on-pump procedure. In a previous study, ${ }^{14}$ we demonstrated that the in-hospital outcomes and the early patency rates of grafts were not different between patients with and without previous PCI who underwent off-pump CABG. However, in that study, the number of patients was small $(\mathrm{N}=545)$, and there were no follow-up results. In our present study, we extended the follow-up to more than 7 years and further demonstrated that long-term survival and freedom from major complications were not affected by previous PCI with stent. Moreover, we adopted propensity score matched-pairs analysis to minimize the difference between the groups, which was not done in our previous study.

In our study, the percentage of use of bilateral internal thoracic artery (ITA) was higher than in other studies. Moreover, there was no difference regarding the use of bilateral ITA between the groups. It is well known that the use of bilateral ITA provides better survival benefit than single ITA. $^{15}$ Furthermore, the combination of off-pump CABG with bilateral ITA grafting also yields favorable outcomes. ${ }^{16} \mathrm{We}$ also demonstrated in a previous study $^{17}$ that off-pump bilateral ITA grafting can be performed safely and effectively with favorable early and 1 -year patency rates in patients with and without left main disease. In our study, the potential influence of bilateral ITA rather than off-pump CABG may have affected the outcome in both groups, although there was no 

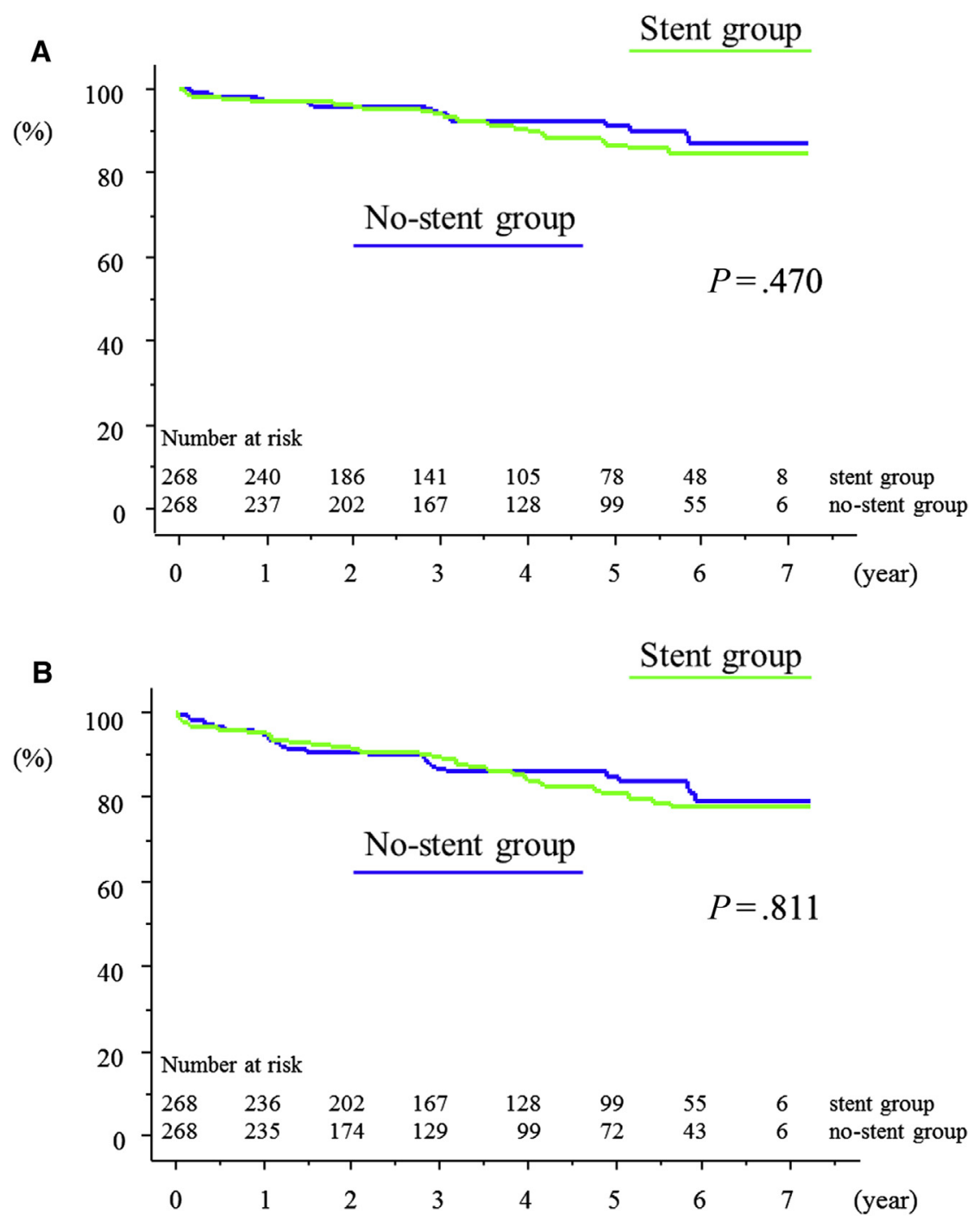

FIGURE 2. A, Kaplan-Meier event-free survival analysis for all deaths in matched patients. B, Kaplan-Meier event-free survival analysis for major adverse cardiac and cerebrovascular events in matched patients.

difference regarding the outcome between the groups. However, because nonuse of bilateral ITA was not associated with late mortality (HR, 1.457; $95 \%$ confidence interval, $0.859-2.472 ; P=.163$ ) and MACCE (HR, 1.101; 95\% confidence interval, $0.708-1.713 ; P=.669$ ) by univariate analysis, further study is necessary to investigate the effect of bilateral ITA in patients with and without previous stents.

Our clinical study has some limitations. The number of patients was relatively small. Although propensitymatched comparison was used, it was still a retrospective observational study and unrecognized confounding factors may exist. We did not show any patency rates of grafts in our study because we did not have enough data on follow-up patency rates of these patients (stent group: 144 out of 268 [53.5\%], no-stent group: 479 out of 897 $[53.4 \%])$.

\section{CONCLUSIONS}

Previous coronary stents do not increase early and long-term morbidity or mortality in patients undergoing off-pump CABG.

The authors thank Ko Bando, MD, for providing an extensive review and valuable comments.

\section{References}

1. Thielmann M, Leyh R, Massoudy P, Neuhäuser M, Aleksic I, Kamler M, et al. Prognostic significance of multiple previous percutaneous coronary interventions in patients undergoing elective coronary artery bypass surgery. Circulation. 2006; 114:I441-7.

2. Hassan A, Buth KJ, Baskett RJ, Ali IS, Maitland A, Sullivan JA, et al. The association between prior percutaneous coronary intervention and short-term outcomes after coronary artery bypass grafting. Am Heart J. 2005; 150:1026-31.

3. Massoudy P, Thielmann M, Lehmann N, Marr A, Kleikamp G, Maleszka A, et al. Impact of prior percutaneous coronary intervention on the outcome of coronary 
artery bypass surgery: a multicenter analysis. J Thorac Cardiovasc Surg. 2009;137: 840-5.

4. Mannacio V, Di Tommaso L, De Amicis V, Lucchetti V, Pepino P, Musumeci F, et al. Previous percutaneous coronary interventions increase mortality and morbidity after coronary surgery. Ann Thorac Surg. 2012;93:1956-62.

5. Gomes WJ, Buffolo E. Coronary stenting and inflammation: implications for further surgical and medical treatment. Ann Thorac Surg. 2006;81:1918-25.

6. Gomes WJ, Giannotti-Filho O, Paez RP, Hossne NA Jr, Catani R, Buffolo E. Coronary artery and myocardial inflammatory reaction induced by intracoronary stent. Ann Thorac Surg. 2003;76:1528-32.

7. Levy JH, Tanaka KA. Inflammatory response to cardiopulmonary bypass. Ann Thorac Surg. 2003;75:S715-20.

8. Fukui T, Tabata M, Manabe S, Shimokawa T, Takanashi S. Graft selection and one-year patency rates in patients undergoing coronary artery bypass grafting. Ann Thorac Surg. 2010;89:1901-5.

9. Fukui T, Tabata M, Taguri M, Manabe S, Morita S, Takanashi S. Extensive reconstruction of the left anterior descending coronary artery with an internal thoracic artery graft. Ann Thorac Surg. 2011;91:445-51.

10. Barakate MS, Hemli JM, Hughes CF, Bannon PG, Horton MD. Coronary artery bypass grafting $(\mathrm{CABG})$ after initially successful percutaneous transluminal coronary angioplasty (PTCA): a review of 17 years experience. Eur J Cardiothorac Surg. 2003;23:179-86
11. van den Brule JM, Noyez L, Verheugt FW. Risk of coronary surgery for hospital and early morbidity and mortality after initially successful percutaneous intervention. Interact Cardiovasc Thorac Surg. 2005;4:96-100.

12. Boening A, Niemann B, Wiedemann A, Roth P, Bödeker RH, Scheibelhut C, et al. Coronary stenting before coronary artery bypass graft surgery in diabetic patients does not increase the perioperative risk of surgery. J Thorac Cardiovasc Surg. 2011;142:e53-7.

13. Lazar HL. Detrimental effects of coronary stenting on subsequent coronary artery bypass surgery: is there another flag on the field? J Thorac Cardiovasc Surg. 2009;138:276-7.

14. Fukui T, Manabe S, Shimokawa T, Takanashi S. The influence of previous percutaneous coronary intervention in patients undergoing off-pump coronary artery bypass grafting. Ann Thorac Cardiovasc Surg. 2010;16:99-104.

15. Taggart DP, D'Amico R, Altman DG. Effect of arterial revascularization on survival: a systematic review of studies comparing bilateral and single internal mammary arteries. Lancet. 2001;358:870-5.

16. Kim KB, Cho KR, Chang WI, Lim C, Ham BM, Kim YL. Bilateral skeletonized internal thoracic artery graftings in off-pump coronary artery bypass: early resul of Y versus in situ grafts. Ann Thorac Surg. 2002;74:S1371-6.

17. Fukui T, Tabata M, Manabe S, Shimokawa T, Shimizu J, Morita S, et al. Offpump bilateral internal thoracic artery grafting in patients with left main disease. J Thorac Cardiovasc Surg. 2010;140:1040-5. 